\title{
Contrôle capillaroscopique sous-unguéal des personnels radio-exposés : résultats préliminaires et incidence en radioprotection
}

\author{
B. PERDEREAU*, F. BRIXY**, R. PENNAROLA***, \\ C. GAURON**, J.M. COSSET*
}

(Manuscrit reçu le 11 janvier 2000, accepté le 12 juin 2000)

RÉSUMÉ Le réseau capillaire cutané de la région sous-unguéale représente un indicateur de grande sensibilité aux altérations provoquées par les rayonnements ionisants. Les auteurs proposent d'améliorer la méthode d'observation capillaroscopique pour assurer la surveillance des mains du personnel exposé professionnellement à de faibles doses répétées durant de nombreuses années au long d'une carrière. Les résultats obtenus au cours de ce travail conduisent à proposer un protocole de contrôle simple à mettre en ceuvre, peu coûteux et d'une totale innocuité, pour la surveillance radiopathologique. L'exploitation des renseignements fournis par l'observation percutanée utilise l'analyse numérisée sur 10 critères, pour augmenter la sensibilité de la technique (capillarométrie). Une analyse plus performante devient en effet nécessaire pour répondre aux nouvelles exigences en matière de radioprotection en application des directives européennes dérivées de la publication 60 de la CIPR. Elle est nécessaire pour analyser les retentissements tardifs des doses cumulées au niveau des mains, cibles principales des irradiations professionnelles (services de médecine nucléaire, de curiethérapie, les laboratoires biomédicaux, et plus encore les équipes d'imagerie interventionnelle). L'exploitation des profils multiparamétriques permet d'éliminer les artéfacts liés à l'âge ou aux diverses pathologies classiques à retentissement microvasculaire, et de dégager les caractères les plus significatifs. L'application de la méthode à 19 sujets exposés professionnellement à de faibles doses en milieu hospitalier a révélé qu'après de longues années d'activité, et en dehors de tout accident de surexposition, les mains présentaient pour 10 sur 19 d'entre eux des anomalies microvasculaires évoquant des conséquences de l'exposition aux radiations ionisantes, et même pour 4 d'entre eux $(20 \%$ de l'échantillon étudié) des caractères évidents de pathologie radique chronique.

ABSTRACT Nailfold capillaroscopic monitoring of radioexposed workers for radioprotection: preliminary results.

The cutaneous capillary network of the nailfold region represents a very sensitive marker of alterations induced by ionising radiation. The authors propose to use this vascular characteristic to ensure surveillance of the hands of workers occupationally exposed to repeated low doses of radiation over many years throughout their career. On the basis of the results of this study, they propose an easy-to-use, inexpensive,

\footnotetext{
* Institut Curie, département Oncologie, Radiothérapie - Radiopathologie clinique, 26 rue d'Ulm, 75248 Paris, France.

** Institut Curie, Médecine du travail, 26 rue d'Ulm, 75248 Paris, France.

*** Facolta Medicina e Chirurgia Medicina del Lavoro, Radiopatologia, Napoli, Italie.
} 
and safe method of radiopathological monitoring. Application of the data provided by percutaneous capillaroscopy, with quantitative analysis of 10 criteria, increases the sensitivity of the technique (capillarometry). A more detailed analysis is necessary to meet the new radioprotection requirements in application of European directives based on CIPR publication 60 . This more detailed analysis is necessary to analyse the late repercussions of cumulative doses to the hands, the main targets at workplace irradiation (nuclear medicine, brachytherapy departments, biomedical laboratories, and especially interventional imaging teams). The low cost of the method constitutes an additional advantage allowing it to be proposed for the occupational healthcare surveillance of exposed workers, especially their hands. Application of this method to 19 subjects occupationally exposed to low doses in hospital revealed that, many years of radioexposition in this environment, in the absence of any overexposure, one half of hands presented abnormalities suggestive of chronic effects of ionising radiation and 4 subjects ( $20 \%$ of the study population) presented obvious features of chronic radiation damage.

\section{Introduction}

Le lit unguéal offre une disposition anatomique particulièrement favorable à l'observation du réseau microvasculaire sous cutané (Carpentier et Franco, 1981; Rinaldi, 1958; Zweifach, 1961). Cette particularité anatomique a été le point de départ des premières observations à des fins médicales dès le début de ce siècle (Lombard, 1912).

Entre 1920 et 1930, Niekau (1923) puis Turano (1930) ont appliqué la capillaroscopie à l'observation directe in vivo des effets des radiations. Malgré l'intérêt indéniable de ces travaux réalisés dans des conditions techniques difficiles, ils n'ont véritablement pris leur essor qu'à partir des années 1970, à la suite de l'introduction d'innovations technologiques et de la dissémination des procédures à risques (développement de la médecine nucléaire et multiplication de l'imagerie radiologique); ainsi, Lenz et al. (1978), Frau et al. (1985) et Pennarola et al. (1983, 1984, 1991) ont établi une véritable séméiologie capillaroscopique des effets des irradiations aiguës accidentelles.

L'extension de la méthode à la surveillance des effets des faibles doses d'irradiation, cumulées sur de nombreuses années, pourraient présenter un intérêt majeur, car aucune technique à ce jour ne permet d'assurer un contrôle facile et fiable de l'exposition des mains des travailleurs du secteur hospitalier (service de médecine nucléaire, de curiethérapie, radiologie interventionnelle, et les laboratoires de recherches biomédicales) ou de l'industrie (gammagraphie, cristallographie, laboratoires). Par ailleurs, la totale innocuité de la méthode d'observation transcutanée des capillaires, associée à la modicité des investissements nécessaires et de son coût de fonctionnement, devraient constituer des arguments favorables à l'extension de la capillaroscopie en radioprotection.

Les auteurs proposent donc ici, d'utiliser la radiosensibilité des capillaires pour assurer la surveillance des travailleurs exposés à de faibles doses d'irradiation 
chronique au niveau des mains. L'observation visuelle capillaroscopique se heurte cependant à son caractère analogique et subjectif. De plus l'absence de signes anatomiques spécifiques des altérations microvasculaires, comme leur relative discrétion, rendent nécessaire l'analyse multiparamétrique des caractéristiques des capillaires sous unguéaux. Celle-ci doit fiabiliser l'information et améliorer les performances de la méthode, elle peut en outre rechercher les concordances spécifiques des anomalies.

Le gain de sensibilité espéré devrait permettre ainsi de disposer d'un outil performant pour assurer le suivi médicoprofessionnel des personnes exposées au niveau des mains, mais aussi d'assurer un contrôle objectif de l'application des nouvelles normes européennes en matière de radioprotection (Euratom, 1997) et en corollaire de justifier la mise en ouvre des dispositions légales pour les cas présentant des signes d'altérations microvasculaires d'exposition excessive aux radiations ionisantes (Delanian, 1993 ; Lenz, 1978).

\section{Rappel général : radiopathologie des capillaires}

L'unité anatomo-fonctionnelle capillaire est constituée de 3 éléments (Fig. 1), selon Chambers et Zweifach (1947):

1. Le canal préférentiel ou axial qui relie l'artériole et la veinule en constituant une sorte de «by-pass » commandé par une gaine musculaire contractile dans sa partie proximale (méta-artériole).

2. Les capillaires vrais naissent de ce canal préférentiel à la sortie de la méta-artériole et se terminent dans la portion distale de ce canal par un «bec de flûte». Leur longueur est très variable et adaptée aux conditions fonctionnelles (échanges gazeux et nutritifs) locales.

3. Des anastomoses directes entre artérioles et veinules ( «shunts directs») dont la signification est controversée (canal de Suquet).

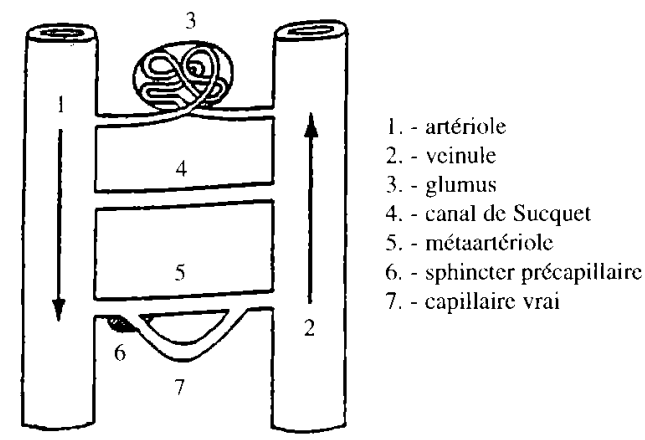

Fig. I. - Schéma fonctionnel de la microcirculation cutanée d'après Chambers et Zweifach (1947). Seul le capillaire vrai (7) est observé et analysé lors d'une observation capillaroscopique de la région sous-unguéale.

Functional pattern of cutaneous microvascularization unit according to Chambers and Zweifach (1947). Only a " true capillary loop " may be observed and analyzed during capillaroscopic examination. 
L'importance fonctionnelle du réseau capillaire est fondamentale car il représente l'ultime ramification de la circulation sanguine à travers laquelle s'effectuent les échanges vitaux vers les tissus (Carpentier, 1984 ; Zweifach, 1973). Il est constitué d'un tubule dont la paroi est constituée d'une couche monocellulaire de nature endothéliale prolongée vers la lumière du tube par une basale surmontée d'un film de fibrine. Celleci est riche en péricytes (cellules de Rouget) dont les sécrétions jouent un rôle essentiel dans les conséquences vasculaires et tissulaires locales des radiations ionisantes.

Au niveau sous-unguéal, c'est essentiellement le capillaire vrai qui est observé ; il représente la seule fraction de la microvascularisation réellement observable directement in vivo à travers la peau (Merlen, 1980).

Dans les conditions normales les capillaires du lit de l'ongle se présentent sous la forme d'anses disposées en épingles à cheveux parallèles ou obliques au plan d'observation du microscope (Fig. 2) (à l'encontre du reste du corps pour lequel les capillaires sont dans un plan perpendiculaire). Ils sont disposés en plusieurs rangées superposées au dessus du réseau sous-papillaire, dont 3 ou 4 sont accessibles à l'observation microscopique chez le sujet normal (Fig. 3).

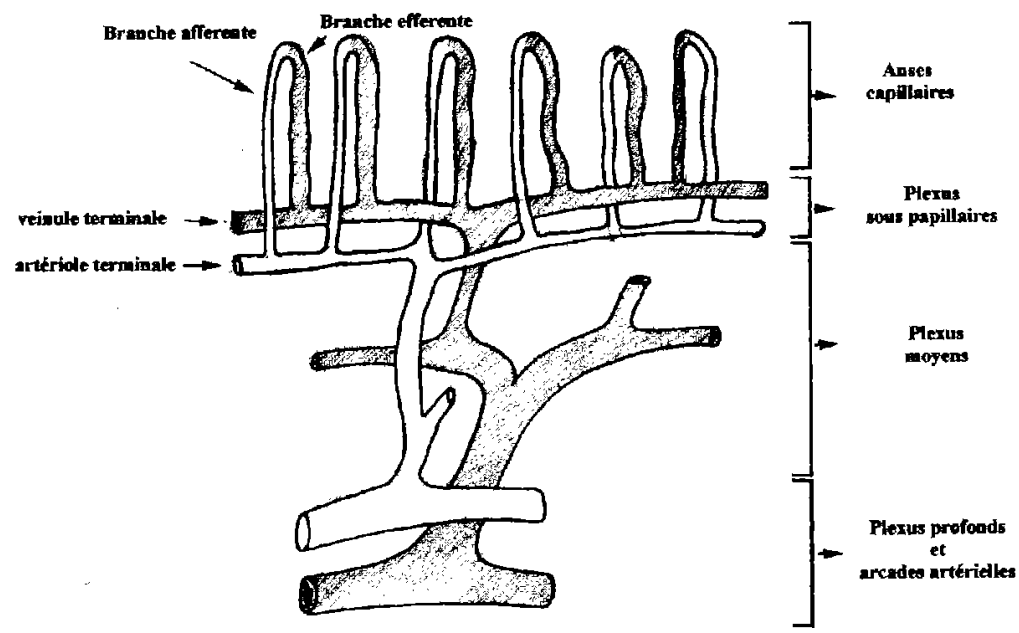

Fig. 2. - Schéma anatomique de la disposition des capillaires vrais ou anses capillaires des régions sous-unguéales.

Anatomical chart of capillary loops of naifolds area. 

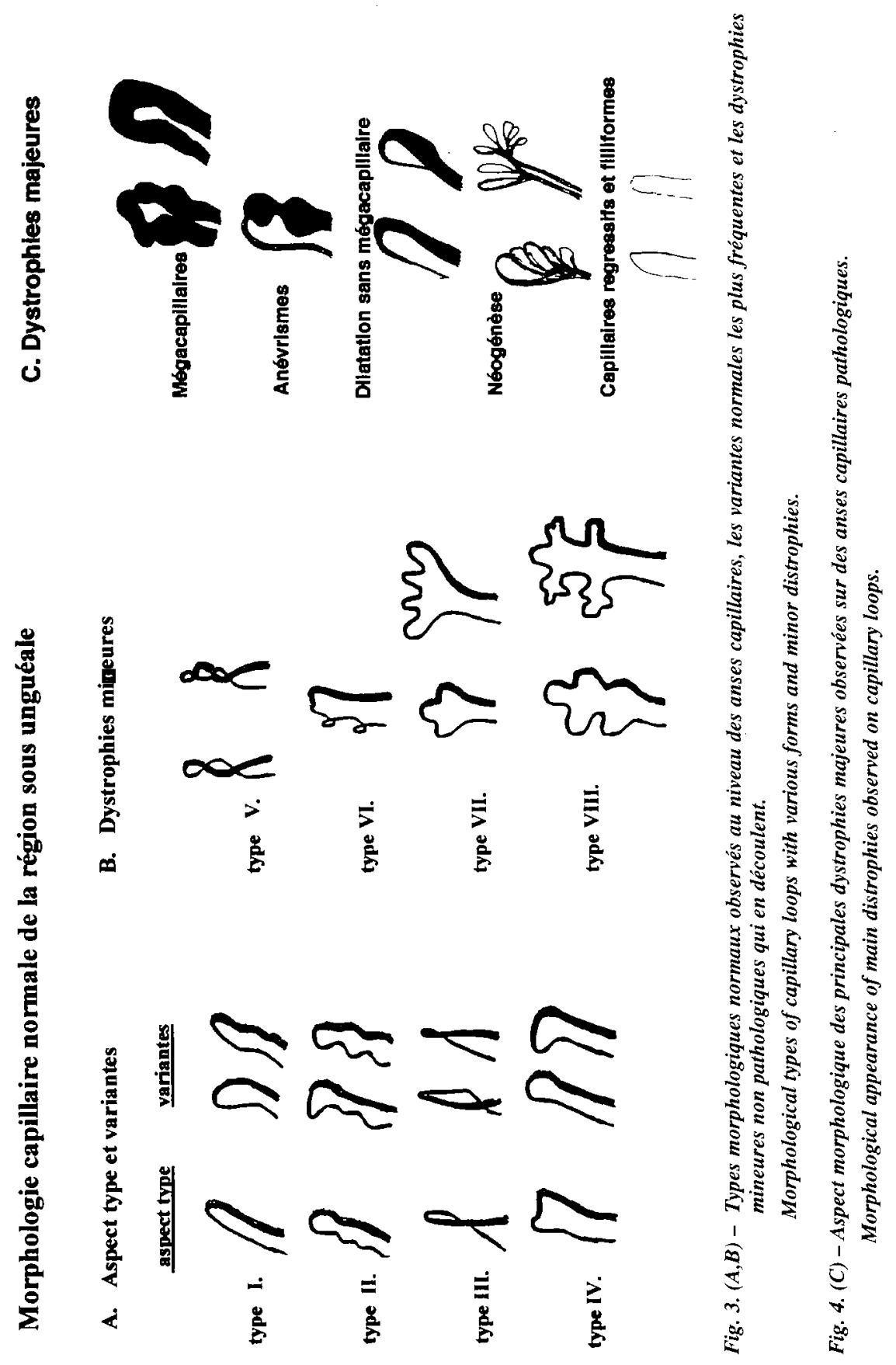


\section{B. PERDEREAU et al.}

Les capillaires, constitués exclusivement d'un endothélium, sont particulièrement sensibles aux radiations ionisantes. Ils constituent des témoins privilégiés de l'exposition radique des doigts (Turano, 1930).

Les diverses altérations microvasculaires se traduisent par des anomalies anatomofonctionnelles (Davis, 1966) incluant une extravasation (œdème) accompagnées d'une réduction de la lumière du capillaire (aspect régressif et filiforme) voire sa disparition (diminution du nombre des anses et zones désertiques) et la présence paradoxale de télangiectasies et de microanévrismes (Fig. 4). Le nombre et l'importance de ces altérations sont liés bien entendu à la dose totale reçue, mais aussi à son fractionnement. La présence de cellules de Rouget, sécrétrices de médiateurs inflammatoires, accentue les effets observés y compris au niveau des tissus avoisinants pour lesquels subsistent un état prolifératif des fibroblastes, une dégradation de la matrice extracellulaire et un infiltrat persistant (Zweifach, 1961; Delanian et al., 1993).

- Si l'irradiation est aiguë, ces processus pathologiques sont graduels, et leur observation séquentielle constitue un dosimètre biologique imprécis mais souvent précieux après un accident. Ils ne dépendent pratiquement, en effet, que de la dose reçue.

- Si l'irradiation est chronique, ses effets peuvent se traduire par des anomalies anatomovasculaires touchant l'intégrité des vaisseaux et leur fonction circulatoire. Moins spectaculaires, plus hétérogènes, ils dépendent largement du fractionnement.

Des publications récentes ont ainsi conduit Lenz et al. (1978) sur 70 sujets et Frau et al. (1985) à décrire des anomalies capillaroscopiques sur 41 sujets pour des doses chroniques ne dépassant pas $10 \mathrm{~Gy}(10 \mathrm{~Sv})$ réparties sur quelques mois (10 à $30 \mathrm{mSv}$ par jour). Pennarola (1983) appliquera la même méthode à titre systématique dans le cadre de la médecine du travail aux travailleurs exposés professionnellement aux radiations ionisantes en dehors de toute irradiation accidentelle aiguë.

Les publications de ces auteurs décrivent un ensemble d'anomalies retrouvées constamment chez les victimes d'irradiation chronique. Ce sont essentiellement : - une réduction du nombre de capillaires avec réduction de leur calibre et allongement de leur parcours, quelquefois compensée par une néogenèse tardive;

- une désorganisation de la distribution;

- une réduction de la marge;

- la présence constante d'un œedème;

- la présence d'ectasies.

L'ensemble de ces caractères traduit un aspect hétérogène des champs d'observation, strictement localisés aux zones exposées (Tab. I).

Aucune anomalie n'est véritablement spécifique de l'atteinte radique et seule la présence simultanée de plusieurs d'entre elles renforce l'hypothèse concernant l'origine des lésions (Fig. 5). 


\section{TABLEAU I}

Principales anomalies pouvant affecter les anses capillaires et leur fonctionnement. Main abnormalities of capillary loops.

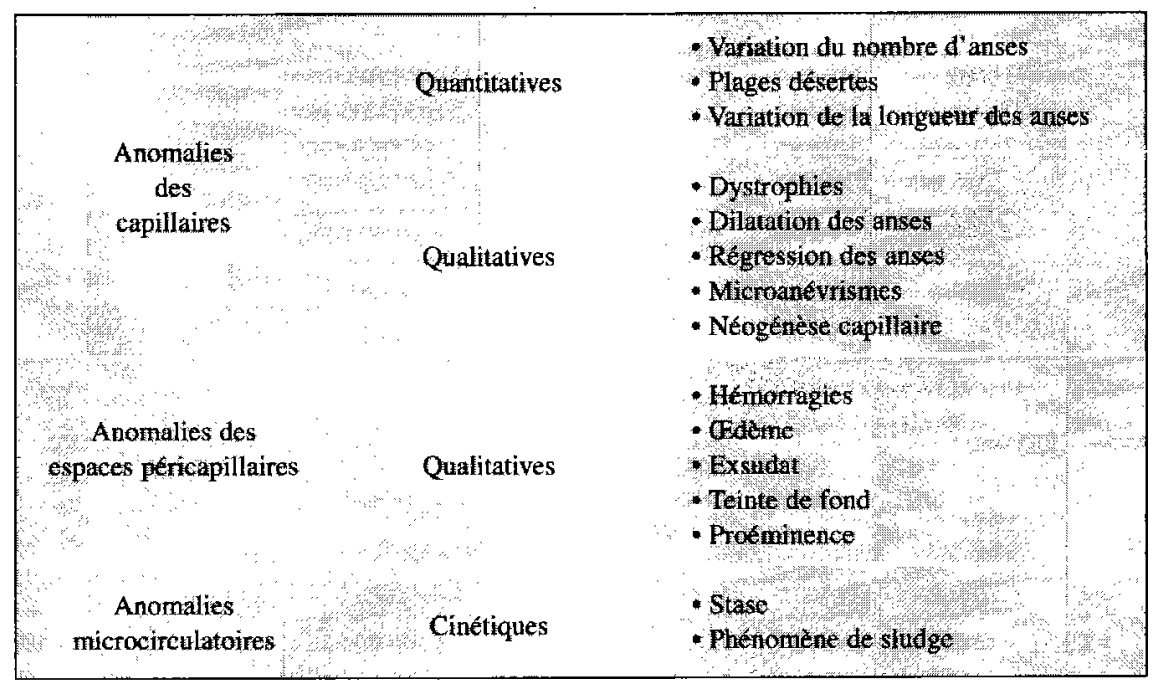

1. Collagenoses: $\quad$ - nombre d'anses réduit ; (sclérodermie généralisée) - œdème diffus et massif; (dermatomyosité) (connectivités) - dystrophies mineures généralisées et spécialement mégacapillaires. (intoxication par polyvinyl)

2. Angiopathies organiques : - capillaires regressifs généralisés.

3. Maladie variqueuse : - télangiectasies généralisées.

4. Maladie de Raynaud : $\quad$ - anses filiformes nombreuses, largement réparties, associées à une paleur de fond.

5. Diabète : - anévrismes fréquents.

6. Acrocyanose : - fond sombre.

7. Lupus : - allongement considérable des anses capillaires.

Fig. 5. - Retentissement microvasculaire de diverses pathologies. La distribution des anomalies morphologiques ou fonctionnelles est toujours généralisée à l'ensemble du réseau capillaire à l'inverse des séquelles d'irradiation strictement localisées aux zones exposées.

Microvascular attack of varied pathology out of radiation effects : morphological or functionnal damager become widespread to capillary network. 


\section{B. PERDEREAU et al.}

\section{Matériel et méthodes}

L'observation des capillaires sous cutanés requiert l'utilisation d'un microscope, d'un éclairage puissant et la transparisation de la peau (Davis, 1966).

\subsection{Le matériel d'observation (Fig. 6)}

\section{Le microscope optique}

Théoriquement, tout microscope offrant une gamme de grossissements moyens (10 à 100) peut servir de capillaroscope (Harders, 1964; Willnow, 1973). Cependant pour observer un champ cutané dans de bonnes conditions techniques, il est préférable de

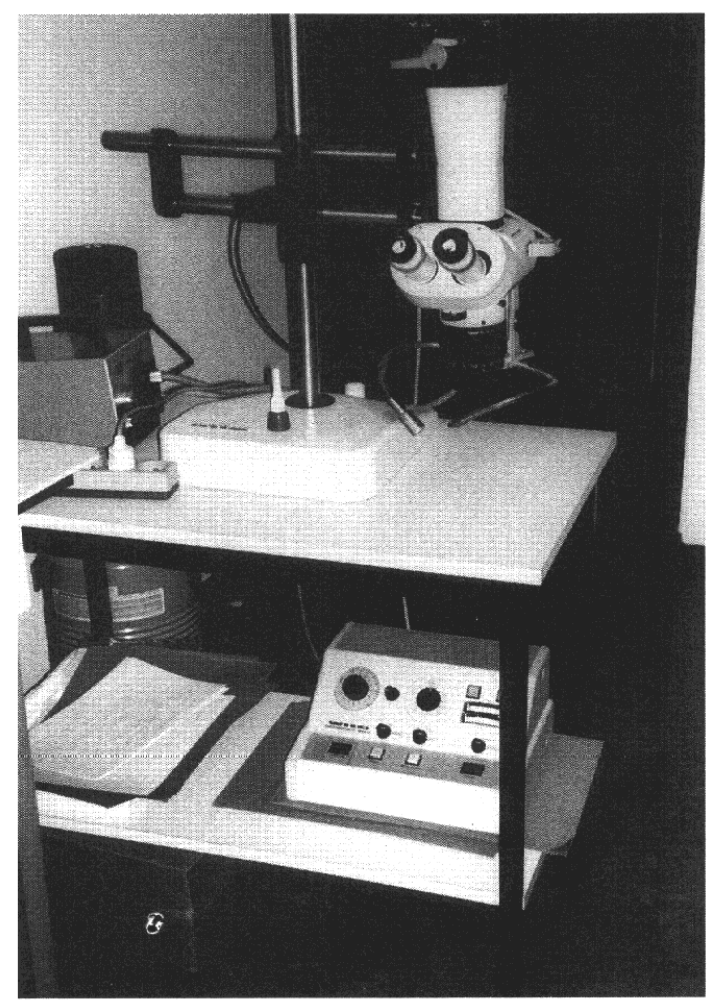

Fig. 6. - Ensemble du matériel nécessaire à l'observation capillaroscopique : le stéréomicroscope Wild M400 avec son équipement photographique, son unité de commande et les fibres optiques souples fixées sur le chariot mobile du corps du microscope.

Capillaroscopic gear needed for cutaneous microvascularization live observation: Wild M400 photo stereomicroscope, control unit and optical fibers light. 
disposer d'un objectif à focale variable (zoom) et de grande distance frontale. Pour conserver une trace de l'observation, le microscope doit être équipé d'une sortie photographique ou vidéo intégrée, il doit disposer d'une ouverture relative de l'objectif (luminosité) aussi grande que possible pour autoriser des vitesses d'obturation rapides, capables d'arrêter les mouvements physiologiques et involontaires du sujet. Nous utilisons un stéréomicroscope Wild M400 dont les grossissements variables de 9 à 50 suffisent largement en pratique. Un réseau micrométrique intégré à l'un des oculaires permet d'effectuer les mesures directes au cours de l'observation visuelle.

\section{L'éclairage}

Un éclairage puissant est indispensable, mais il ne doit pas surchauffer le champ d'observation. Des fibres optiques souples, indépendantes du microscope répondent au mieux à cet impératif. Notre installation est constituée d'un générateur constitué d'une lampe halogène de $15 \mathrm{~V}-150 \mathrm{~W}$ avec diaphragme. Les fibres (2 ou plus) sont munies de focalisateurs optiques amovibles pour concentrer le maximum de lumière sur une surface très restreinte.

\section{Le statif}

Un statif de table, lourd, permettant le décentrement important du microscope est indispensable.

\section{La transparence}

La visualisation transcutanée des capillaires n'est possible qu'après transparisation de la couche kératinisée superficielle. Tous les observateurs utilisent actuellement des huiles d'immersion synthétiques totalement atoxiques dont l'indice de réfraction est voisin de celui de l'objectif, sa viscosité est suffisante pour se maintenir sur le rebord unguéal le temps nécessaire à l'examen.

\section{Le centrage}

Afin de garantir l'immobilité maximale des doigts, le poignet doit reposer sur la table d'observation et le doigt examiné doit être sur un support plat muni d'une dépression de positionnement d'une largeur et longueur d'un doigt. Ce support doit garder une mobilité précise sur les 2 axes pour centrer et déplacer la zone à observer.

\subsection{Les sujets observés}

Les sujets sont volontaires, ils ont été exposés professionnellement en milieu hospitalier depuis de nombreuses années, et sont observés dans le cadre de la radioprotection (Tab. II). 
TABLEAU II

Caractéristiques de l'échantillon des sujets observés. Characteristics of patients.

\begin{tabular}{|c|c|c|c|c|c|c|c|}
\hline Ident. & $\begin{array}{l}\text { Identification } \\
\text { Sexe }\end{array}$ & âge & $\begin{array}{c}\text { Habitus } \\
\text { Tabac/alcool }\end{array}$ & $\begin{array}{l}\text { Pathol. } \\
\text { Vase. Ete. }\end{array}$ & Durée & $\begin{array}{c}\text { Exposition } \\
\text { Origine }\end{array}$ & Nature \\
\hline 1 & F & 43 & Non & Non & 21 & Med. Nuc. & $\gamma$ \\
\hline 2 & F & 50 & Non & Non & 28 & Med. Nuc. & $\gamma$ \\
\hline 3 & M & 53 & Non & Non & 22 & Med. Nuc. & $\gamma$ \\
\hline 4 & M & 65 & Non & Non & 13 & Med. Nuc. & $\gamma$ \\
\hline 5 & F & 48 & Non & Non & 13 & Med. Nuc. & $\gamma$ \\
\hline 6 & M & 28 & Non & Non & 3 & Med. Nuc. & $\gamma$ \\
\hline 7 & F & 60 & Oui & Non & 27 & Labo & $\mathrm{x}$ \\
\hline 8 & F & 58 & Non & Non & 36 & Med. Nuc. & $\gamma$ \\
\hline 9 & F & 44 & Non & Non & 22 & Med. Nuc. & $\gamma$ \\
\hline 10 & F & 24 & Non & Non & 3 & Med. Nuc. & $\gamma$ \\
\hline 11 & M & 61 & Non & Non & 21 & Labo & $\mathrm{x}$ \\
\hline 12 & F & 51 & Non & Non & 28 & Curiethér. & $\beta$ \\
\hline 13 & $\mathrm{~F}$ & 58 & Non & Non & 30 & Med. Nuc. & $\gamma$ \\
\hline 14 & M & 36 & Non & Non & 13 & Med, Nuc. & $\gamma$ \\
\hline 15 & M & 69 & Non & Non & 26 & Med. Nuc. & $\gamma$ \\
\hline 16 & M & 57 & Non & Non & 25 & Med. Nuc. & $\gamma$ \\
\hline 17 & F & 73 & Non & Non & 26 & Radio & $\mathrm{x}$ \\
\hline 18 & F & 80 & Non & Non & 20 & Radio & $\mathrm{x}$ \\
\hline 19 & $\mathrm{M}$ & 53 & Oui & Non & 19 & Maintenance & $\gamma$ \\
\hline
\end{tabular}

Notre échantillon comporte 19 sujets (11 femmes, 8 hommes): 13 en médecine nucléaire dont 7 femmes; 2 en laboratoire dont 1 femme; 2 femmes en radiodiagnostic; 1 femme en radiothérapie; et enfin un technicien de maintenance. La plupart de ces sujets avait un long passé professionnel en milieu exposé, supérieur à 10 ans.

La répartition entre hommes et femmes, comme la connaissance de l'âge moyen et la durée moyenne d'exposition ont été déterminants dans la faible dispersion des 
résultats. L'homogénéité de l'échantillon a permis d'autre part l'exploitation des résultats malgré le nombre restreint de sujets. Ainsi :

- L'âge moyen des sujets observés est de 52,7 \pm 13,5 ans (limites d'âge de la population: $24-80)$ avec la répartition suivante :

$-53,5 \pm 14$ ans $(24-80)$ pour les femmes $(n=11)$,

$-51,5 \pm 12$ ans $(28-65)$ pour les hommes $(n=8)$.

- L'ancienneté professionnelle moyenne est de 20,9 $\pm 10,3$ ans (3-36), pour l'ensemble du groupe.

Tous les sujets provenaient de services utilisant les radiations dans le secteur hospitalier ; leur fonction impliquait une exposition majoritaire des mains dont il est difficile d'évaluer correctement les doses reçues. Ces doses étaient inférieures cependant au seuil d'apparition des signes cliniques, à l'exception vraisemblable de l'un deux qui présentait des anomalies cutanées au niveau de la pulpe de $D_{1} D_{2} D_{3}$ de la main droite, pour lesquelles aucun diagnostic dermatologique précis n'avait pu être porté et pour lequel l'origine radique n'était pas exclue.

Aucun d'eux n'a présenté au cours de sa carrière de dépassements des doses tolérées par les normes en vigueur au niveau du film dosimétrique «poitrine», mais en l'absence de contrôle systématique des doigts avec des bagues dosimétriques, d'utilisation encore trop rare et non systématique, malgré la valeur démontrée de la méthode ( 100 services médicaux, scientifiques ou industriels, seulement reçoivent régulièrement de l'OPRI les bagues dosimétriques, [estimation du $1^{\text {er }}$ trimestre 2000]). Il semble hasardeux d'apprécier correctement les doses réellement accumulées par chacun d'eux à ce niveau. Une évaluation arbitraire, établie à partir des données physiques classiques conduit à des doses voisines de $20 \mathrm{~Sv}$ en médecine nucléaire pour 20 années d'exercice, soit $\sim 3 \mathrm{mSv}$ par jour. D'après les évaluations publiées par Batchelor et al. (1991), dans les conditions actuelles de radioprotection, il paraît licite de considérer que les doses reçues à l'époque étaient sensiblement supérieures (protège seringues non généralisés, utilisation courante de radioéléments très énergétiques). Ces doses pourraient être supérieures en curiethérapie, en particulier pour les techniciens manipulant les fils d'iridium.

\subsection{Paramètres de l'observation capillaroscopique}

L'étude morphologique d'un champ capillaroscopique permet d'obtenir des données quantitatives et qualitatives sur les différentes caractéristiques de l'image et ses éventuelles anomalies. L'analyse multiparamétrique des images capillaroscopiques a nécessité la sélection de 10 paramètres à partir des caractéristiques analogiques de l'observation pour leur apport en radiopathologie :

1 - Nombre de rangées visibles : trois ou quatre rangées sont normalement visibles.

La présence d'un œdème, ou d'une hyperkératose, peut limiter la visibilité d'une ou plusieurs rangées. 
2 - Longueur des anses capillaires $\left(\mathrm{N}^{\mathrm{al}}=300-350 \mu \mathrm{m}\right)$ : l'allongement des anses capillaires est caractéristique de leur difficulté à assurer leur fonction d'échanges métaboliques.

3 - Couleur de fond : elle traduit la qualité globale de la microvascularisation profonde. Tout changement de couleur est étroitement lié à la cinétique circulatoire dans la zone observée. Un fond pâle correspond à une diminution de remplissage des plexus ; un fond pourpre ou violacé est caractéristique d'une stase.

4 - Marge sous unguéale $\left(\mathrm{N}^{\mathrm{al}}=200-300 \mu \mathrm{m}\right)$ : Elle résulte de la longueur des capillaires et de la présence d'une extravasation.

5 - Nombre d'anses capillaires observables par $\mathrm{mm}\left(\mathrm{N}^{\mathrm{al}}=13-17\right)$ : la diminution, voire la disparition des anses capillaires sur un nombre de doigts réduits de la main préférentielle, sont des signes d'atteinte microvasculaire majeure.

6 - Néogenèse capillaire : adaptation microvasculaire à une atteinte modérée chronique.

7 - Distribution des capillaires : chez le sujet normal, la distribution est homogène et régulière. Toute irrégularité est suspecte, mais les petits traumatismes provoqués par les travaux manuels, bien localisés, peuvent gêner l'interprétation.

8 - Caractères morphologiques : un certain nombre de dystrophies sont bien systématisées (Figs. 4B, 5 et 6). Normalement, on trouve moins de 10\% de dystrophies mineures. Elles augmentent légèrement avec l'âge, mais toutes les dystrophies majeures sont pathologiques.

9 - Rapport des diamètres artériolo-veinulaire $\left(\mathrm{N}^{\mathrm{al}}=1,2-1,3\right)$ : c'est un bon indice de souffrance des différents segments constituant l'anse capillaire.

10 - Circulation capillaire : normalement rythmée et régulière (pouls capillaire), elle est tributaire des altérations subies par les capillaires.

L'examen doit être précédé d'une enquête minutieuse sur le passé professionnel, l'environnement, et les habitudes de vie (Fig. 7). L'âge est un facteur important car il retentit naturellement sur l'aspect et la fonction circulatoire, il en est de même de certains troubles métaboliques (dyslipidémie, diabète, uricémie,...) ou de pathologies vasculaires (cardiopathies, hypertension, pathologies vasculaires) ou périphériques (acrocyanose, maladie de Raynaud). Enfin l'arthrite rhumatoïde et le psoriasis sont susceptibles (et la liste n'est pas limitative) de provoquer des altérations des capillaires, dont il est nécessaire de tenir compte avant toute interprétation. La généralisation à l'ensemble des doigts des anomalies microvasculaires pathologiques suffit en général pour éviter toute confusion avec une origine radique (toujours localisée) (Maricq et al., 1976; Merlen, 1979; Potten, 1985; Vayssariat et Priollet 1983).

Pour un même sujet, chaque paramètre est mesuré sur chaque doigt (Tab. III).

\subsection{Conduite pratique de l'examen capillaroscopique}

Le patient dont les doigts sont examinés est assis confortablement face à l'opérateur sur un siège réglable permettant de placer le plan d'observation à la hauteur du cœur pour annuler les différences de pression sanguine liées à l'orthostatisme. 


\section{QUESTIONNAIRE}

\section{IDENTIFICATION :}

. Nom :

. Prénom :

. âge :

. Sexe :

\section{HABITUS :}

. Tabac:

. Alcool :

. Onychophagie :

. Manucure :

- Act. manuelle:

. Loisirs :

\section{BIOLOGIE :}
. Cholestérol :
. Glycémie :
. Ac. urique :
.P. A. :

\section{PATHOLOGIE :}

- Diabète :

. Pathol. bronchique :

. Mal. endocrinienne :
. Rhumatisme :

. Psoriasis :

. Vasculaire :

\section{PROFESSION :}

- Secteur d'activité

- Catégorie professionnelle :

. Exposition radiologique: . Nature :

. Durée :

- Type de rayonnement :

. Dosimètre poitrine : . Dosimètre doigt :

. Dépassement des normes :

Fig. 7.- Modèle du questionnaire préalable à tout examen capillaroscopique sous-unguéal. Prerequisite questionnaire before nailfold capillaroscopic examination. 
TABLEAU III

Modèle de la grille de report des valeurs mesurées sur chacun des doigts observés.

Grid of direct observation values.

\begin{tabular}{|l|c|c|c|c|c|c|c|c|c|c|c|}
\multicolumn{1}{c|}{} & Normal & D1d & D2d & D3d & D4d & D5d & D1g & D2g & D3g & D4g & D5g \\
\hline & No à 4 & & & & & & & & & & \\
\hline Long. anses & 300 à 350 & & & & & & & & & & \\
\hline Couleur fond & Code :4 & & & & & & & & & & \\
\hline Marge & 200 à 300 & & & & & & & & & & \\
\hline Nb anses & 13 à 17 & & & & & & & & & & \\
\hline Néogénèse & 0 & & & & & & & & & & \\
\hline Distribution & Code : 3 & & & & & & & & & & \\
\hline Morphologie & Code : 3 & & & & & & & & & & \\
\hline D. eff. / D. aff. & 1,2 à 1,3 & & & & & & & & & & \\
\hline Circulation & Code : 3 & & & & & & & & & & \\
\hline
\end{tabular}

Pour assurer l'immobilité indispensable des doigts, le patient repose la main ainsi que l'avant bras sur la table d'examen, le doigt examiné repose librement sur une gouttière aménagée pour que la base de l'ongle soit perpendiculaire à l'axe optique.

Une goutte d'huile d'immersion est déposée sur le rebord unguéal et les fibres optiques sont dirigées et focalisées (lorsqu'elles possèdent une optique terminale) sur le champ d'observation. Un repérage de la région est réalisé à faible grossissement $(\times 10)$ pour apprécier l'hétérogénéité, la distribution, l'éventuelle présence d'hémorragies et anévrismes.

Les dix doigts sont examinés systématiquement. Une observation est consignée pour chacun d'entre eux et des documents photographiques ou vidéo sont enregistrés simultanément.

Tous les capillaires ne sont pas situés dans le même plan, mais seule la première rangée est prise en compte lors du dénombrement. 


\subsubsection{Examen du sujet normal}

Dans les conditions normales chez le sujet jeune sans aucune des pathologies précitées, les capillaires apparaissent, au niveau sous-unguéal, sous la forme d'anses disposées en épingles à cheveu généralement parallèles au plan focal (sauf pour les travailleurs manuels, chez lesquels les anses capillaires sont plus ou moins redressées) disposées en plusieurs couches individualisables (3 à 4) au-dessus du réseau vasculaire sous papillaire observable lorsque la transparence du milieu est excellente (Fig. 8).

Chaque rangée de capillaires est régulièrement disposée, le fond est rose et transparent. Les capillaires doivent apparaître isolés, de types rectilignes ou variants (I à IV), comprenant éventuellement un petit contingent (10\% max.) de formes particulières, de types légèrement dystrophiques (V à VIII). Leur disposition est régulière et homogène, leur calibre est fin et le diamètre du segment efférent est légèrement supérieur au segment afférent (Fig. 9). Le flux sanguin est parfaitement visible le long des capillaires soulignant le caractère discontinu de la vasomotricité microvasculaire. La présence d'un halo autour des anses capillaires est un caractère peu fréquent mais non pathologique.

\subsubsection{Examen du sujet irradié}

La conduite de l'examen est identique à celle du sujet normal. On ajoute habituellement des microphotos de la peau au niveau de la zone d'irradiation et des ongles (si nécessaire) des doigts concernés.

\section{Exploitation des données}

La variabilité habituelle de la plupart des caractères descriptifs des capillaires ne permet qu'exceptionnellement de se satisfaire de la présence d'une altération observée sur un seul paramètre pour affirmer la réalité d'une lésion, et plus rarement encore de son origine radique.

Cette difficulté d'interprétation nécessite la validation des altérations décrites en recourant à des témoins d'une part et en s'affranchissant des artéfacts d'autre part. Ce travail a porté sur une série historique de sujets dont les doigts non exposés ont servi de témoins. La possibilité d'utiliser le sujet comme son propre témoin résulte de l'étroite localisation de l'exposition professionnelle chronique aux seuls doigts manipulateurs (doigts de la pince de la main préférentielle) comme l'ont montré Batchelor et al. (1991). Ces auteurs ont calculé un gradient d'exposition voisin de 10 entre $\mathrm{D}_{2}$ exposé et $\mathrm{D}_{5}$ non exposé pour la main préférentielle. Ce gradient est plus élevé encore pour la main symétrique. Les doigts non exposés, à savoir $\mathrm{D}_{4}$ et $\mathrm{D}_{5}$ des 2 mains ont été comparés entre eux, pour justifier leur valeur de référence : leur observation ne présente pas de différence significative (test $t$ de Student $=0,43$ 


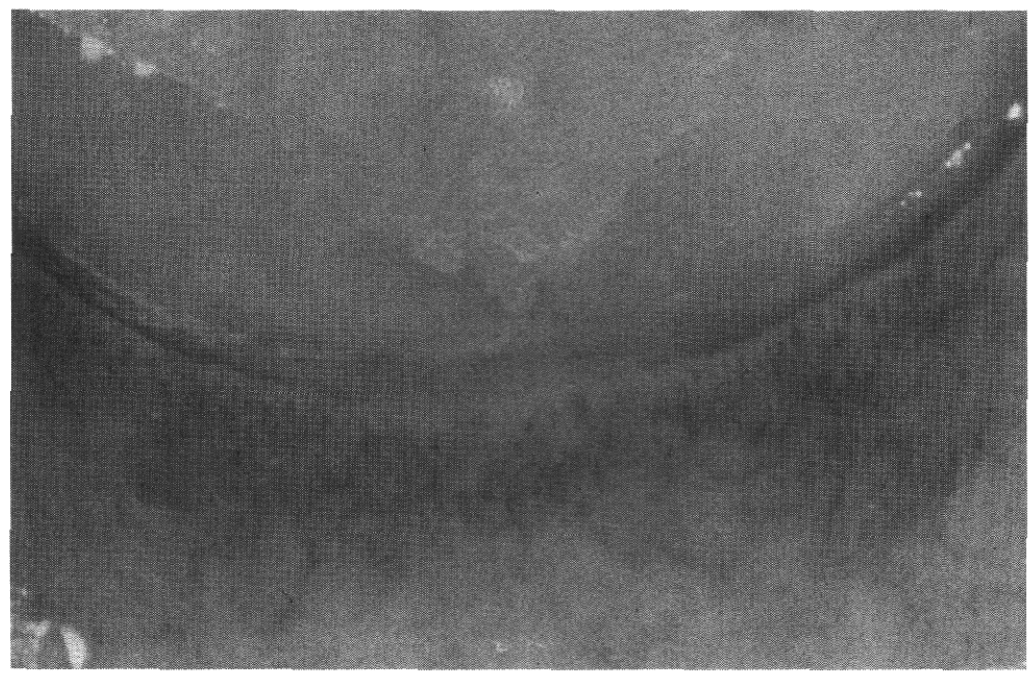

Fig. 8. - Observation à faible grossissement $(G=6)$ de la totalité de la région sous-unguéale de D3 droit. Le grossissement est insuffisant pour analyser la morphologie capillaire, mais elle permet d'étudier la distribution et la visibilité éventuelle du réseau sous papillaire. Ce type de document permet en outre de comprendre le principe de l'observation capillaroscopique.

Examination of F3r (right finger 3) nailfold. The low magnification (x 6) shows the distribution of capillary but not morphological features.

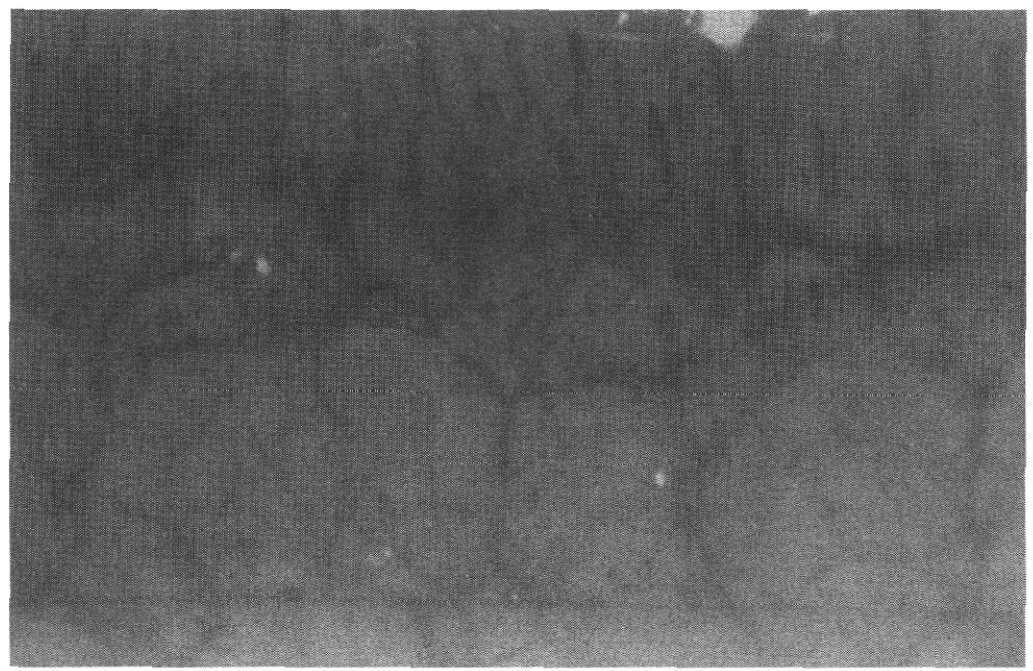

Fig. 9. - Capillaroscopie d'un sujet normal à grossissement d'analyse $(G=32)$. La transparence est bonne, trois rangées de capillaires normaux (type I essentiellement) dont les caractéristiques sont normales (nombre, longueur, diamètre), ainsi que la couleur de fond.

Capillaroscopic examination of normal patient ( $x$ 32). The transparency is good, three rows of capillary are visible (type I). Other datas are normal. 
pour $20 \mathrm{DL}, p=0,667$, NS). Le nombre d'anomalies relevées est très faible : main préférentielle $D_{4}=10, D_{5}=5$ et main symétrique $D_{4}=6, D_{5}=5$ pour les 19 patients, soit moins d'une anomalie observable par sujet ( 0,3 en moyenne). Ces résultats ne sont pas incompatibles avec ceux publiés par Vayssariat $(1983,<2$ en moyenne pour la main d'une population normale) et notre expérience antérieure ne montre pas de différence significative ( 0,5 anomalie par doigt).

L'éventuelle présence d'artefacts doit impérativement être prise en compte pour valider toute réponse :

- Les artefacts d'origine pathologique ne sont pas rares, mais leur répartition généralisée à l'ensemble des doigts des deux mains permet de les éliminer d'emblée, comme la concordance des altérations ; ils ne faussent pas l'interprétation mais peuvent éventuellement réduire la sensibilité en cas de gros retentissements microvasculaires. Aucun sujet de la série observée ne présentait d'artéfacts d'origine pathologique ou traumatique.

- Des artéfacts dus à l'âge se traduisent par de discrètes dystrophies à la fois caractéristiques et généralisées, ne perturbant pas l'analyse capillaroscopique. Ils sont fréquents après 55 ans ( 6 sur 9 des plus de 55 ans, contre 1 sur 10 pour les moins de 55 ans [Chi2 avec correction Yates $=4,328, p=0,038$ ]). La comparaison des résultats obtenus sur les doigts non exposés avec ceux observés sur 10 sujets d'âge analogue, provenant de nos archives, ne montrent pas de différence significative (test t de Student $=0,68, p=0,412$, NS).

- La comparaison systématique aux doigts témoins non exposés élimine de fait les anomalies généralisées (test $t$ de Student $=0,51$ pour $10 \mathrm{DL}, p=0,631$, NS).

- Seule une intense activité manuelle localisée peut entraver sérieusement l'observation (hyperkératinisation gênant l'observation et remaniements microvasculaires), d'autant qu'elle se situe habituellement au niveau des doigts les plus exposés. Ce phénomène est cependant assez rare parmi la population exposée chroniquement à des sources d'irradiation.

L'observation microvasculaire d'une zone limitée à la région exposée ne retiendra donc que des anomalies ponctuelles localisées aux zones les plus exposées.

Trois critères anatomofonctionnels ont été retenus pour caractériser les effets des rayonnements sur la structure des capillaires:

1. l'extravasation, marquée localement par la présence d'un œdème permanent; 2. les dystrophies morphologiques et les modifications qui affectent la distribution des capillaires;

3. les changements observés dans la dynamique circulatoire locale.

En pratique, lors d'un examen capillaroscopique, la présence d'un œedème est caractérisée par :

- le nombre de rangées de capillaires visibles (paramètre 1) ;

- la longueur des anses capillaires (paramètre 2) ;

- la couleur de fond (paramètre 3) ;

- la marge unguéale (paramètre 4). 
L'atteinte microvasculaire est attestée par:

- le nombre d'anses capillaires par mm, hors néogenèse (paramètre 5);

- la présence d'une néogenèse capillaire (paramètre 6);

- la distribution des capillaires y compris la présence de plages désertes (paramètre 7);

- le type morphologique dominant (paramètre 8 ).

La cinétique sanguine :

- le rapport des diamètres entre les branches capillaires éfférentes et afférentes (paramètre 9);

- la circulation observée (paramètre 10).

Pour être exploitées numériquement, les variables qualitatives doivent être codifiées ; en l'absence d'une référence reconnue, les auteurs utilisent une échelle basée sur les observations de Merlen, Vassayrat et Pennarola plus particulièrement:

- couleur de fond : $\quad$ code de 1 à 5 .

- distribution: $\quad$ code de 1 à 5 .

- morphologie: $\quad$ code de 1 à 5 .

- circulation sanguine: code de 1 à 4 .

Quant aux variables quantitatives, elles sont exprimées directement dans leur système d'unité ou sans unité pour les rapports. La présence d'une néogenèse, par ailleurs, est comptabilisée en nombre de «bouquets par $\mathrm{mm} »$.

\section{Résultats}

Les informations recueillies au cours de l'examen capillaroscopique concernent les 10 paramètres définis au niveau des espaces sous-unguéaux de chacun des dix doigts pour les 19 sujets de l'étude.

Les résultats bruts font apparaître un nombre d'altérations plus élevé au niveau de certains doigts. L'histogramme de leur distribution montre une nette prédominance des doigts de la pince $\left(\mathrm{D}_{1} \mathrm{D}_{2} \mathrm{D}_{3}\right)$ de la main préférentielle et plus spécialement $\mathrm{D}_{2}$ (reconnu 11 sur 19). Un premier test $t$ séries appariées confirme que la main préférentielle est significativement la plus atteinte $(t=5,29$ pour $18 \mathrm{DL}$, $p<0,001)$; un second test $t$ séries appariées confirme que la pince est significativement plus atteinte que les doigts les moins exposés $(t=9,81$ pour $18 \mathrm{DL}$, $p=0,007)$. Pour être considérées comme significatives, les dystrophies devront porter sur plus de $10 \%$ des capillaires observés par champs (seuil habituel de signification en pathologie microvasculaire) après élimination des artéfacts (origine professionnelle, environnementale ou pathologique). Les figures 10 , 11, et 12 sont des exemples de différents types d'anomalies sur les documents photographiques directs. 


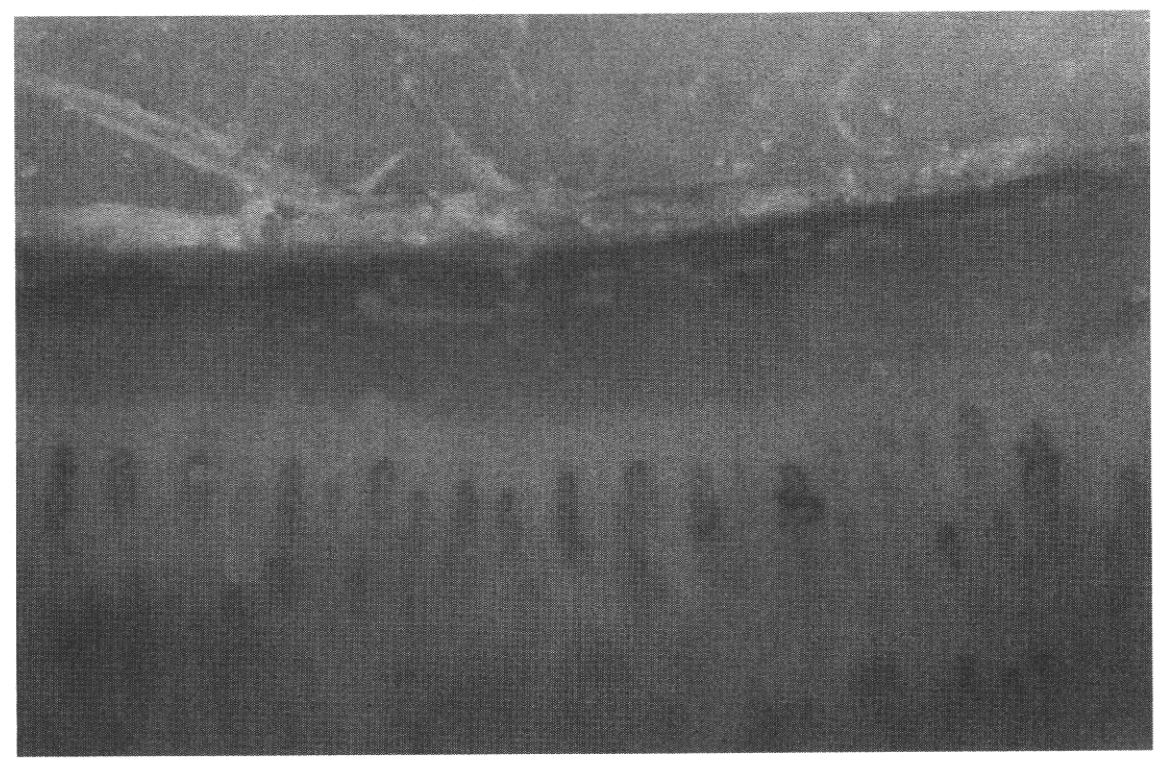

\begin{tabular}{|c|c|c|c|}
\hline Nombre de rangées visibles & 2 & Néogenèse & 0 \\
\hline Longueur des anses & 320 & Distribution & Irrégulière \\
\hline Fond & orange & Morphologie & $\begin{array}{l}\text { Dystrophies } \\
\text { mineures }\end{array}$ \\
\hline Marge & 300 & $\mathrm{D}$ eff. / $\mathrm{D}$ aff. & 1,6 \\
\hline Nombre d'anses par mm & 11 & circulation & sludge \\
\hline
\end{tabular}

Chez une femme de 45 ans, technicienne de médecine nucléaire (cas n ${ }^{\circ} 5$ ). Les anomalies discrètes touchent la distribution. À noter les nombreuses dystrophies mineures et la présence d'un léger œè̀me.

Fig. 10. - Altérations capillaroscopiques : $1^{\text {er }}$ exemple $(G=32)$. Capillaroscopic abnormalities: 1 st example (case $N^{\circ} 5$ ). 


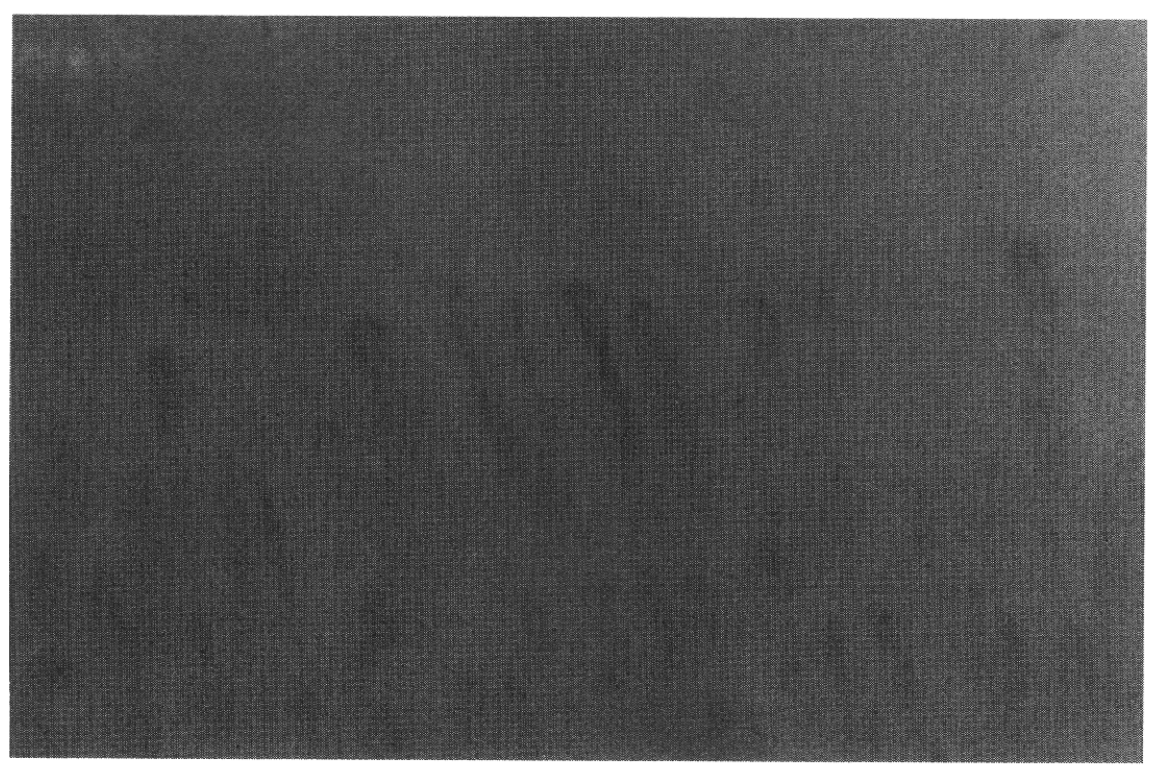

\begin{tabular}{|lc|cc|}
\hline Nombre de rangées visibles & 1 & Néogenèse & 7 \\
\hline Longueur des anses & 400 & Distribution & Irrégulière \\
\hline Fond & pâle & Morphologie & Dystrophies \\
& & & mineures \\
\hline Marge & 105 & D eff. / D aff. & 2,3 \\
\hline Nombre d'anses par mun & 9 & circulation & sludge \\
\hline
\end{tabular}

Chez une femme de 73 ans ancienne manipulatrice en radiologie ayant pratiqué durant de nombreuses années de radioscopie ( $\operatorname{cas} \mathrm{n}^{\circ} 17$ ). Les anomalies importantes touchent la totalité des paramètres et sont évocatrices de lésions microvasculaires d'origine radique. À noter l'important œedème, le faible nombre d'anses, la dilatation des anses efferentes et la présence d'une zone désertique.

Fig. 11. - Altérations capillaroscopiques : $2^{e}$ exemple $(G=32)$.

Capillaroscopic abnormalities: 2nd example (case $\left.N^{\circ} 17\right)$. 


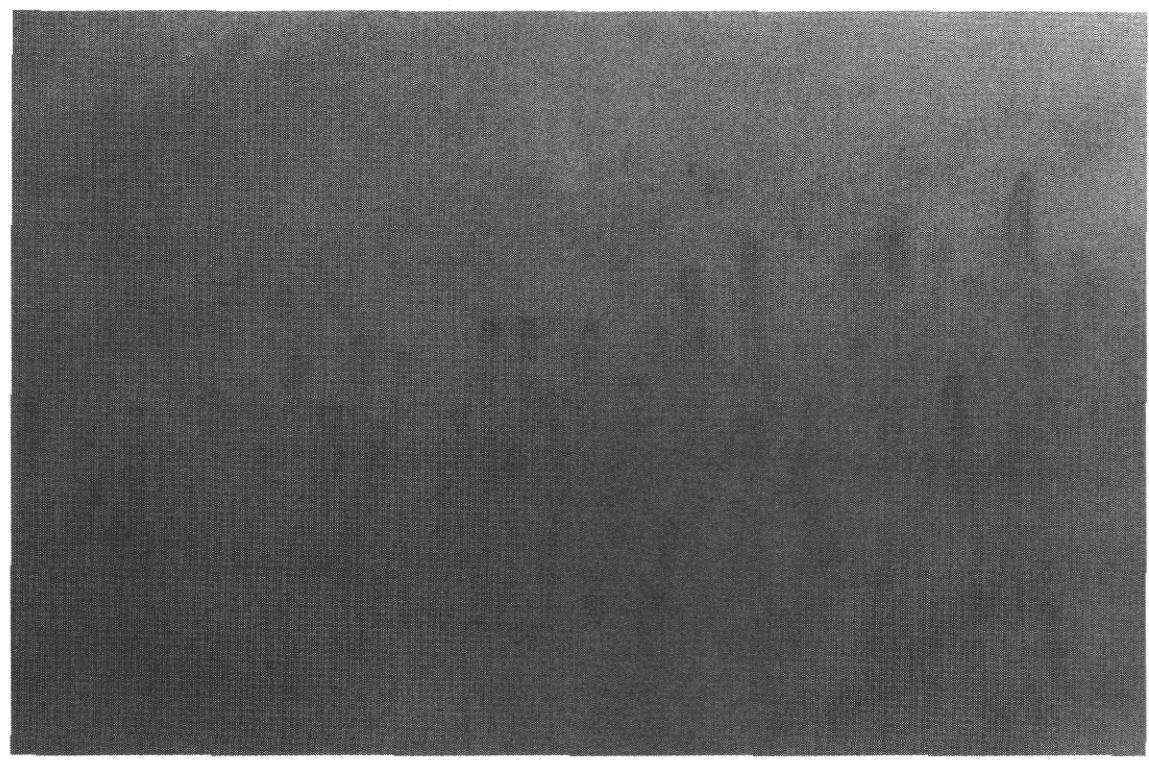

\begin{tabular}{|lc|cc|}
\hline Nombre de rangées visibles & 1 & Néogenèse & 8 \\
\hline Longueur des anses & 360 & Distribution & Irrégulière \\
\hline Fond & orange & Morphologie & $\begin{array}{c}\text { Dystrophies } \\
\text { mineures }\end{array}$ \\
\hline Marge & 100 & D eff. / D aff. & 1,4 \\
\hline Nombre d'anses par mim & 11 & circulation & sludlge \\
\hline
\end{tabular}

Chez une femme de 60 ans (cas $n^{\circ} 7$ ). Les importantes anomalies touchent tous les paramètres et sont évocatrices de lésions microvasculaires d'origine radique. À noter un œdème, l'aspect regressif des anses capillaires par ailleurs sans dystrophies importantes, le faible nombre d'anses, la dilatation des anses efferentes et la présence d'une zone désertique.

Fig. 12. - Altérations capillaroscopiques : $3^{e}$ exemple $(G=32)$.

Capillaroscopic abnormalities: 3 rd example (case $N^{\circ}$ ). 
TABLEAU IV

Valeurs numériques mesurées ou calculées sur la région présentant le plus grand nombre de perturbations microvasculaires pour l'ensemble des dix paramètres des dix-neuf sujets de l'échantillon.

Data measured or calculated on the most disturbed nailfold capillaroscopic zona from ten fingers.

\begin{tabular}{|c|c|c|c|c|c|c|c|c|c|c|}
\hline Ident & Rangs & Long. & Fond* & Marge & Anses & Néocap. & Distri. & Morph* & D.efo.af & Circul \\
\hline 1 & 4 & 350 & 3 & 310 & 16 & 0 & 4 & 3 & 1,4 & 3 \\
\hline 2 & 2 & 230 & 3 & 90 & 10 & 0 & 1 & 1 & 1,6 & 2 \\
\hline 3 & 2 & 300 & 1 & 280 & 15 & 1 & 4 & 3 & 2 & 2 \\
\hline 4 & 4 & 370 & 3 & 190 & 16 & 0 & 3 & 3 & 1,4 & 3 \\
\hline 5 & 2 & 210 & 3 & 300 & 11 & 0 & 2 & 3 & 1,6 & 2 \\
\hline 6 & 3 & 400 & 3 & 110 & 19 & 0 & 2 & 2 & 1,2 & 3 \\
\hline 7 & 1 & 430 & 1 & 100 & 11 & 8 & 4 & 4 & 1,4 & 2 \\
\hline 8 & 2 & 250 & 1 & 210 & 16 & 0 & 4 & 5 & 1,2 & 3 \\
\hline 9 & 3 & 410 & 3 & 110 & 18 & 0 & 3 & 2 & 1,5 & 3 \\
\hline 10 & 2 & 300 & 2 & 190 & 13 & 1 & 2 & 4 & 1,3 & 2 \\
\hline 11 & 3 & 350 & 2 & 200 & 16 & 2 & 3 & 2 & 1,6 & 3 \\
\hline 12 & 2 & 400 & 1 & 100 & 12 & 5 & 4 & 5 & 2,5 & 2 \\
\hline 13 & 2 & 380 & 1 & 110 & 10 & 1 & 5 & 5 & 1,8 & 2 \\
\hline 14 & 4 & 300 & 3 & 100 & 12 & 0 & 3 & 3 & 1,3 & 3 \\
\hline 15 & 2 & 410 & 2 & 310 & 19 & 0 & 5 & 3 & $2 ; 3$ & 2 \\
\hline 16 & 5 & 400 & 2 & 110 & 18 & 0 & 3 & 2 & 1,3 & 3 \\
\hline 17 & 1 & 400 & 1 & 105 & 9 & 7 & 4 & 4 & 2,3 & 2 \\
\hline 18 & 2 & 420 & 1 & 100 & 12 & 0 & 4 & 5 & 1,8 & 2 \\
\hline 19 & 3 & 350 & 3 & 300 & 16 & 0 & 3 & 3 & 1,4 & 3 \\
\hline
\end{tabular}

L'ensemble des résultats est reporté dans le tableau général (Tab. IV). Pour faciliter la compréhension, celui-ci est scindé en 3 tableaux partiels dont chacun correspond à l'un des critères sélectionnés.

\section{Présence d'un oedème local (Tab. V.A.)}

Le nombre de rangées de capillaires effectivement visible dépend directement de la présence et de l'importance d'un infiltrat qui augmente le flou de l'observation.

La couleur de fond constitue un paramètre complémentaire important. Par ailleurs la chronicité d'un œdème aboutit à un allongement des anses capillaires. Elle se 
traduit en outre par une modification de la marge unguéale dont la hauteur est tributaire de la longueur des anses capillaires.

Cinq sujets présentent des perturbations au niveau des 4 paramètres (cas 7,12 , 13,17 et 18), à l'inverse 4 sujets présentent un aspect normal pour ces mêmes 4 paramètres (cas $1,4,5,19)$, les autres ne présentant que des anomalies ne concernant qu'un seul paramètre (cas $6,11,14$ ) ou deux (cas 2, 3, 8, 9, 10).

\section{Les remaniements vasculaires (Tab. V.B.)}

Les modifications anatomiques concernent en premier lieu le nombre des anses individualisables par unité de longueur $(\mathrm{mm})$ sur une seule rangée, leur diminution comme les modifications de leur morphologie ou de leur distribution sont autant de signes d'une souffrance microvasculaire. Enfin la présence d'une néogénèse souligne l'importance de l'atteinte fonctionnelle.

Quatre sujets présentent des perturbations sur l'ensemble des quatre paramètres (cas 7, 12, 13, 17). Cinq ne présentent aucune modification (cas 4, 5, 14, 16, 19). Les autres présentent des anomalies limitées. Huit cas présentent un processus de néogénèse plus ou moins accentué. Chez 3 d'entre eux (cas 7, 12, 17), le nombre de néocapillaires est important $\left(\mathrm{N} \geq 5 \mathrm{~mm}^{-1}\right)$.

\section{La cinétique vasculaire (Tab. V.C.)}

L'état fonctionnel des vaisseaux se définit selon l'observation de la circulation sanguine.

Neuf sujets observés présentent des anomalies circulatoires sur les 2 paramètres (cas $1,2,3,7,12,13,15,17,18$ ). Dix ont une cinétique normale (cas 4, 6, 8, 9, $14,16,19)$, ou subnormale (cas $5,10,11$ ).

Sur la confrontation des résultats provenant des trois tableaux des critères (Tab. V.D) :

- un seul sujet présente des altérations ne touchant qu'un seul critère (mais validé par la présence simultanée de trois paramètres modifiés) (cas 16);

- trois sujets présentent des altérations touchant deux critères :

- les cas 8 et 9 ont un cedème confirmé,

. le cas 1 des anomalies circulatoires sans cdème, et tous ont des remaniements morphologiques hétérogènes et localisés ;

- neuf sujets $(2,3,7,10,12,13,15,17,18)$ présentent des altérations sur les trois critères retenus, et quatre d'entre eux $(7,12,13,17)$ ont la totalité des paramètres altérés.

Pour ces sujets la présence d'un oedème est quasi constante (12 sur 13) ; il représente l'anomalie la plus fréquente pour cet échantillon; les dystrophies et les hétérogénéités de distribution touchent 11 sur 13, et pour 8 d'entre eux ( 8 sur 11), la néogénèse capillaire représente l'anomalie la plus fréquente. 

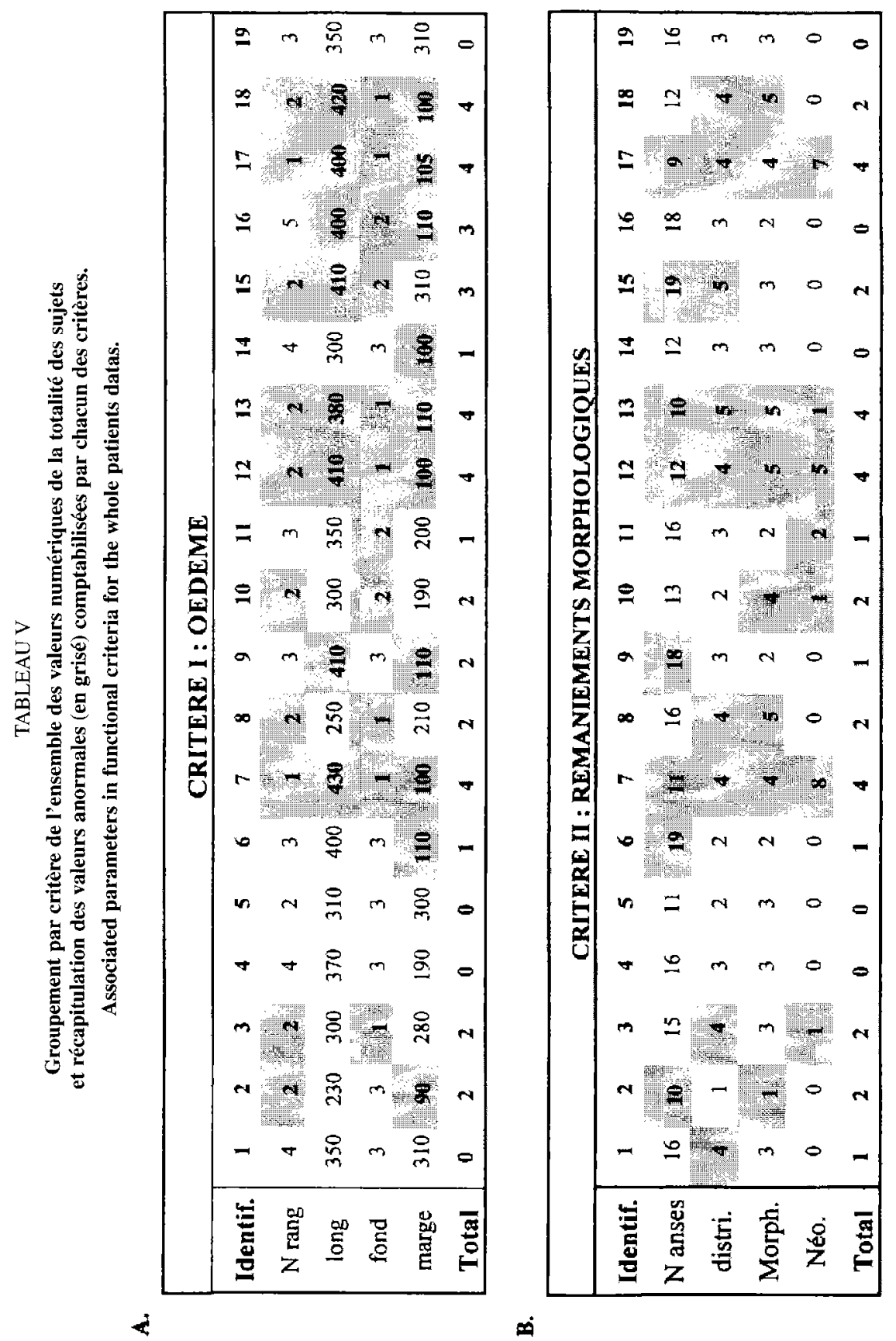


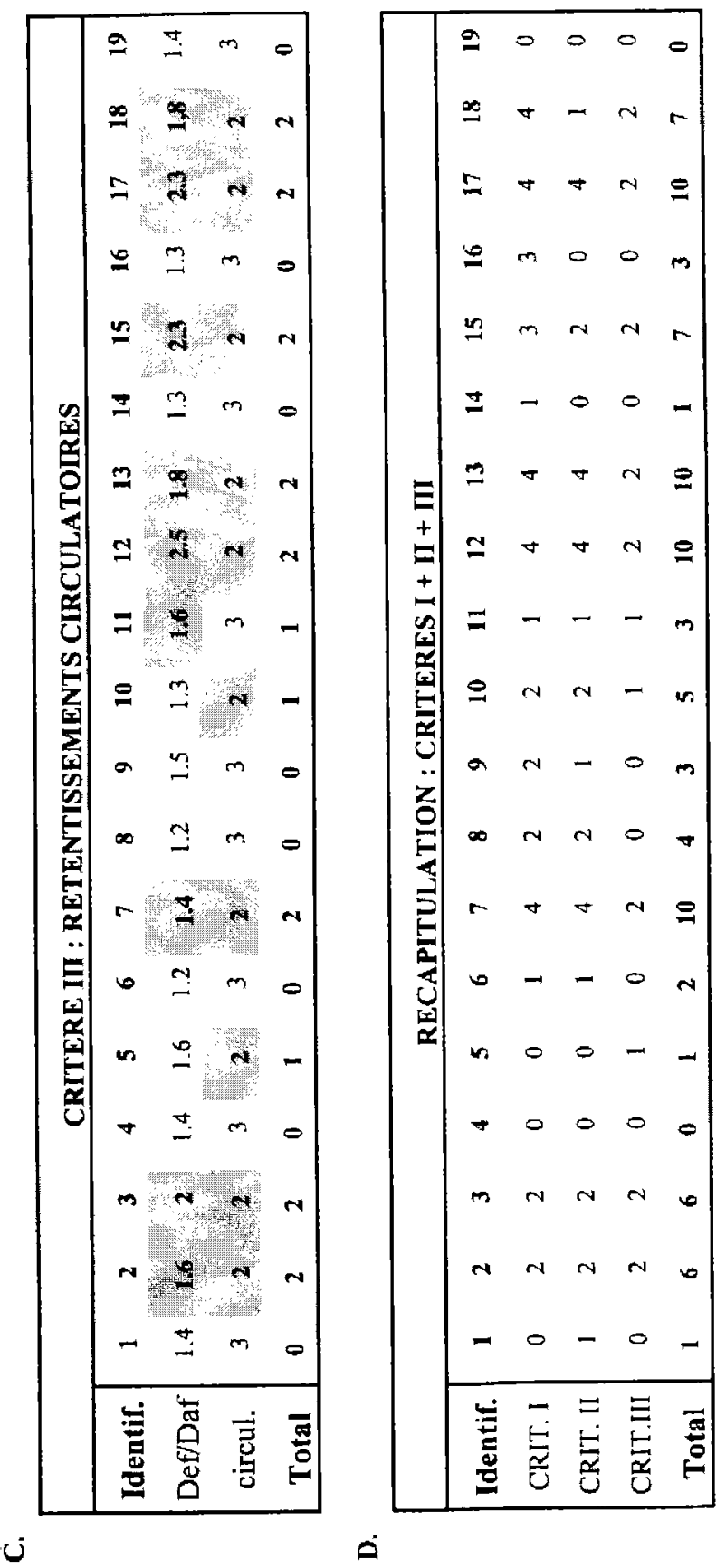




\section{B. PERDEREAU et al}
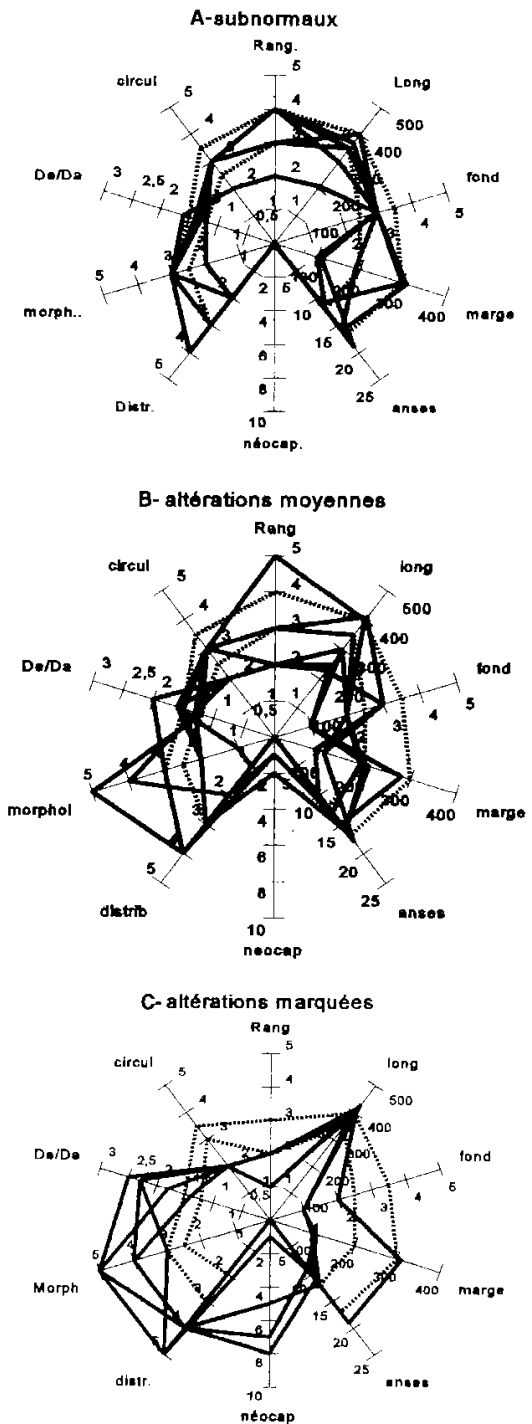

Les sujets ont été regroupés selon l'importance des anomalies microvasculaires : A. subnormaux, B. altérations moyennes, C. altérations importantes. La zone grisée correspond à la variabilité des valeurs normales $(\mathrm{m} \pm \mathrm{sd})$.

Fig. 13. - Comparaison des profils individuels des valeurs mesurées ou calculées sur les dix paramètres à l'aide de profils circulaires.

Comparison of individuals results on 10 parameters spider web, in three levels: A. subnormality, B. low damages, $C$. high damages. 
Sept autres sujets ne présentent pas d'altérations significatives (cas 5, 6, 11 et 14), et deux sujets (cas 4 et 19) peuvent être considérés comme strictement normaux.

La projection des résultats d'un même sujet, sous forme de profils circulaires à partir de données numériques de tous les paramètres analogiques après codage individualisant la réponse, permet ainsi une exploitation individuelle facile, rapide, et une comparaison interindividuelle instantanée.

Les graphiques de la figure 13 montrent l'ensemble de l'exploitation obtenue par cette méthode de traitement pour chacun des 19 sujets observés, avec les valeurs normales prises pour référence pour chaque paramètre à partir de la littérature et de notre expérience antérieure. Les altérations modérées n'induisent pas de profils très caractéristiques (Figs. 13A et 13B) mais il n'en est pas de même pour les sujets présentant des altérations marquées (Fig. 13C) dont les altérations les plus fréquentes se traduisent, sur ce type de graphique, par un profil particulier en forme de triangle dont la pointe serait dirigée vers l'axe «longueur des anses» et la base large serait tournée vers les axes «morphologie, distribution, et la présence de néocapillaires». Cette remarque pourrait accélérer l'analyse multiparamétrique par simple représentation graphique et simplifier le recueil des données statistiques. Le nombre d'anomalies augmentent en fonction de la durée d'exposition (ancienneté professionnelle) comme le montre le tableau VI :

TABLEAU VI

Fréquence des anomalies capillaires en fonction de la durée d'exposition. Capillaroscopic abnormalities rate according to exposure time.

\begin{tabular}{|ccccc|}
\hline & \multicolumn{3}{c|}{ Nombre d'altérations (paramètres modifiés) } \\
\hline Durée exposition & $<4$ & $<7$ & $\geq 7$ & Total \\
\hline$<15$ ans & 4 & 1 & 0 & 5 \\
$<25$ ans & 3 & 2 & 1 & 6 \\
\hline 25 ans & 0 & 3 & 5 & 8 \\
\hline
\end{tabular}

La recherche d'une proportionnalité entre la dose représentée par le temps d'exposition et les remaniements microvasculaires observés, conduit à une courbe dont l'expression mathématique la plus probable est de type logarithmique (Fig. 14) :

$$
\mathrm{Y}=0,816 \mathrm{e}^{(0,064 \mathrm{x})} \text {, coefficient de corrélation }: \mathrm{R}=0,717 \text {. }
$$

La corrélation calculée est insuffisante pour conclure à une dépendance directe. Néanmoins, l'incidence de l'âge étant éliminée par l'analyse, la durée de l'exposition paraît donc logiquement jouer un rôle dans l'accumulation des dommages observés. Cette hypothèse cependant, demanderait à être confortée ultérieurement. 


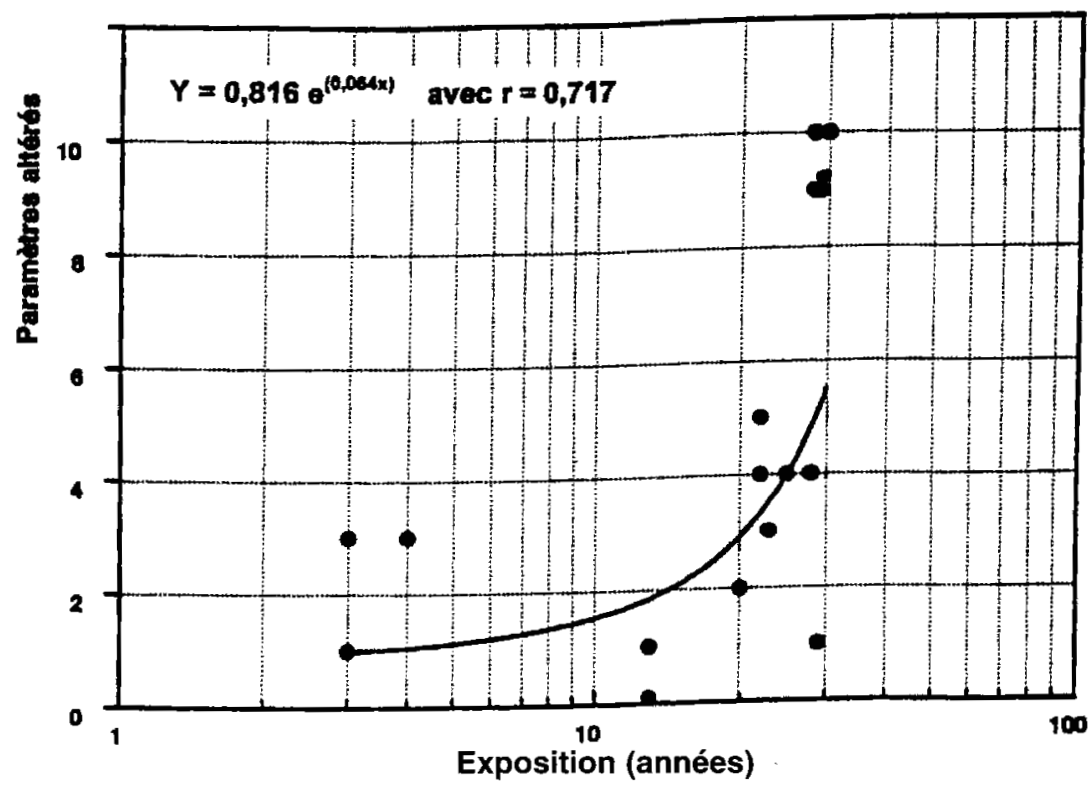

Fig. 14. - Relation entre la durée d'exposition et le nombre d'anomalies capillaires observées.

Graphical analysis of capillaroscopic abnormalities.

\section{Discussion}

L'observation analogique transcutanée des capillaires présente des difficultés de validation et d'interprétation lorsque les altérations sont discrètes, même si le réexamen par un tiers diminue les doutes et les imprécisions. Les données quantitatives, par définition, ne dépendent pratiquement pas de l'observateur (nombre, longueur, diamètre par exemple) à l'inverse des données qualitatives qui, lorsqu'elles sont numérisées, peuvent cependant se soustraire au moins partiellement à la subjectivité de l'observateur (codification des caractères).

Il ne faut pas oublier que le réseau n'est observable que sur des zones privilégiées pour lesquelles les capillaires sont étalés parallèlement à la surface de la peau. Les régions sous-unguéales des doigts et des orteils ainsi que la conjonctive bulbaire représentent les meilleures zones d'observation. Or, les mains représentent la région du corps habituellement la plus exposée professionnellement, et leur atteinte microvasculaire constitue une sorte de «marqueur» des effets biologiques du niveau d'exposition. En dehors de cette disposition anatomique particulièrement favorable, notre travail a été facilité par la sélection de 
notre échantillon de sujets dont l'effectif, bien que faible, présente une bonne homogénéité des conditions d'exposition professionnelle chronique faible. Les 19 sujets observés sont tous issus du milieu hospitalier (y compris le technicien de maintenance), où l'exposition est constituée essentiellement par la manipulation de sources radioactives non scellées destinées à l'imagerie nucléaire, la radiothérapie métabolique ou la recherche biomédicale (17 sur 19). Cette identité de risque présente certaines particularités utiles pour rendre cohérente l'exploitation : l'exposition est faible mais régulière car la préparation journalière de sources radioactives destinées au diagnostic, au traitement ou aux travaux de recherche impose une faible distance entre la source et les doigts du préparateur ou de l'utilisateur, et ceci malgré la présence de matériel de protection. Malgré les faibles activités relatives mises en jeu, l'exposition effective est mal connue et aucune reconstitution dosimétrique confirmée n'a pu malheureusement être réalisée sur de longues périodes au niveau des doigts, et les sujets de notre étude n'y font pas exception.

L'absence de cette information ne nous a pas permis de transformer la courbe durée/effets en courbe dose/effets.

Aucun des sujets ne présentait d'artefacts majeurs dus à des pathologies métaboliques ou vasculaires; les 6 personnes âgées de plus de 60 ans n'étaient atteintes que de dystrophies mineures ne portant que sur un seul paramètre distribué sur la totalité des doigts. Enfin, aucun des sujets de l'étude n'a montré d'anomalies significatives au niveau de régions sous-unguéales autres que celles constituant la pince $\left(D_{1} D_{2} D_{3}\right)$ de la main préférentielle.

Ces résultats confirment largement les anomalies observées classiquement lors des irradiations chroniques, mais leur niveau liminaire et leur caractère de très longue chronicité permettent d'en souligner les particularités telles qu'elles apparaissent sur cette série limitée :

- une hétérogénéité et une irrégularité de distribution qui frappent d'emblée l'observateur;

- des altérations limitées exclusivement au niveau des doigts soumis aux rayonnements ionisants;

- un œdème systématique limité aux zones exposées;

- une hétérogénéité morphologique localisée (altérations antagonistes);

- des plages de raréfaction capillaire localisées (étape ultime de la régression capillaire), sans zones désertiques caractérisées;

- une néovascularisation typique en bouquets.

Les résultats obtenus au cours de ce travail permettent de nous interroger sur la fréquence des séquelles provoquées par l'activité professionnelle en milieu exposé. Notre échantillon est certes particulier puisqu'il ne provient que d'un secteur d'activité et que la moyenne de la durée d'exposition dépasse 20 ans, mais il met 
ainsi en évidence l'élévation du pourcentage des anomalies microvasculaires avec le cumul de la dose totale reçue. Ainsi :

- 9 sujets seulement ne montrent pas d'anomalies significatives des effets des radiations ionisantes (seuls deux d'entre eux présentent un aspect strictement normal), et c'est dans ce groupe que les sujets les plus «jeunes» sont observés (ancienneté professionnelle moyenne : 11,5 ans).

- 10 sujets, soit la moitié de l'échantillon, montrent des anomalies évocatrices d'une atteinte radique, mais avec des degrés variés. Ainsi, parmi ceux-ci, 9 présentent un nombre plus ou moins important d'anomalies cohérentes traduisant l'impact de l'irradiation à un niveau inférieur au stade clinique, mais 4 sujets présentent la totalité des signes caractéristiques d'une irradiation chronique. Dans ce groupe figure le seul sujet dont les signes cutanés étaient apparents mais non déterminants. Ces sujets présentent le premier stade clinique de l'atteinte radique. Ces chiffres peuvent surprendre car de nombreux sujets n'ont pas dépassé la moitié de leur carrière alors que les nouvelles réglementations en matière de radioprotection plus restrictives entreront en vigueur au cours de l'année 2000.

\section{Conclusion}

Jusqu'à présent aucune méthode ne permettait d'observer in vivo les altérations provoquées au niveau des doigts par les radiations ionisantes et plus spécialement par l'exposition chronique faible.

La capillaroscopie multiparamétrique offre une technique simple à mettre en œuvre, pertinente et peu coûteuse qui a été exploitée au cours de ce travail sur un échantillon de 19 professionnels hospitaliers exposés quotidiennement à des doses faibles durant de nombreuses années. Ces sujets ne présentent aucun signe clinique, à l'exception d'un seul, alors que la capillaroscopie multiparamétrique a révélé que plus de la moitié (10 sur 19) d'entre eux cumulaient des altérations microvasculaires sous-unguéales évocatrices de séquelles radiques.

Ce constat doit faire réfléchir car de nombreux risques apparaissent, en particulier dans le domaine médical avec le développement de la radiologie interventionnelle, le regain de la curiethérapie métabolique, le développement de la radio-immunothérapie, et l'arrivée de l'imagerie par positons. Les irradiations ainsi délivrées aux mains peuvent s'accroître non seulement pour les personnels des services exposés (radiologie, radiothérapie, médecine nucléaire) généralement bien informés des mesures de radioprotection, mais également pour de nouveaux personnels (chirurgiens, cardiologues, infirmiers) trop souvent ignorant des dispositions à respecter.

Si ces premières observations se confirmaient elles pourraient conduire à déterminer plusieurs attitudes pratiques en fonction de la gravité des altérations micro- 
circulatoires caractéristiques, strictement localisées aux doigts les plus exposés : - maintien en milieu exposé avec surveillance de routine simple par capillaroscopie pour les sujets ne présentant pas d'anomalies significatives;

- surveillance renforcée avec éventuellement éloignement des zones à risque pour les sujets présentant des signes d'altérations radiques significatifs sans entraîner des signes cliniques cutanés (avec rythme de surveillance double);

- prise en charge radiopathologique et suppression de tout risque d'exposition pour les autres cas présentant un maximum d'altérations significatives et concordantes comportant notamment la présence de néocapillaires en bouquets.

En conséquence la surveillance de routine serait vraisemblablement utile, mais la faiblesse numérique de l'échantillon observé actuellement ne permet pas encore d'en fixer le rythme, néanmoins il semblerait qu'elle ne soit pas nécessaire avant 10 années de pratique professionnelle.

Remerciements: Les auteurs tiennent à remercier particulièrement Bernard Aubert pour ses encouragements et les références dosimétriques qu'il leur a aimablement fournies.

\section{RÉFÉRENCES}

Batchelors S., Penfold A., Aric I., Huggins R. (1991) Radiation dose to the hands in nuclear medicine, Nucl. Med. Comm. 12 (5), 439-44.

Carpentier P., Franco A. (1981) La capillaroscopie périunguénale. Labor. Lafon. Deltacom., Paris.

Carpentier P. (1984) Physiologie et exploitation de la microcirculation et de la vasomotricité, Drugs and Deseases 1 (2), 13-20.

Chambers R., Zweifach B.W. (1947) Blood-borne vasotropic substance in experimental chock, Am.J. Physiol. 150, 239.

Davis E., Landau J. (1966) Clinical Capillary Microscopy. Thomas, Springfield, USA.

Delanian S., Martin M., Housset M. (1993) La fibrose iatrogènique en cancérologie. I. Aspects descriptifs et physiolopathologiques, Bull. Canc. 80 (3), 192-201.

Euratom (1997) Directive Euratom 97/43. J.O. des Communautés européennes 9/7/97, Bruxelles.

Frau P., Meloni M., Bario P., Usala A., Casula D. (1985) Quadro capillaroscopico in sogetti esposti a radiazioni ionizzanti, Riv. Inf. Mal. Prof. 52, 67-72.

Harders H. (1964) The contribution of microscopes to the study of living circulation: clinical biomicroscopy, J. Roy. Microsc. Soc. 83, 45-53.

Lenz U., Schutmann W., Arndt D., Thormann (1978) Late effects of ionizing radiation on the human skin after occupationalexposures. In: Proceedings of the symposium on the late effects of ionizing radiation, 1, pp. 32 I-329. Intern. Atomic Agency, Vienna.

Lombard W.P. (1912) The blood pressure in the arterioles, capillaries and small veins of the human skin, Am. J. Physiol, 29, 335-362.

Maricq H.R., Johnson M.N., Whetstone C.L., Leroy E.C. (1976) Capillary abnormalities in polyvinyl chloride production, Workers J. M. A. 236, 1369-1371.

Merlen J.F. (1979) Acrosyndrômes vasculaires et capillaroscopie, J. Mal. Vasc. 4, 43-45.

Merlen J.F. (1980) La capillaroscopie, moyen d'exploitation fonctionnelle, Ann. Med. Nancy 18 , 189-195. 


\section{B. PERDEREAU et al.}

Niekau B. (1923) Anatomische und Klinische Beobachtungen mit der Hautkapillarmikroskopie, Strahlentherap. 15, 372.

Pennarola R. (1983) L'esame capillaroscopico nella prevenzione delle manifestazioni da radiazioni ionizzanti, Rass. Intern. Cl. Ter. 63, 307-313.

Pennarola R., Gongora R. (1983) Rilievi di capillaroscopia in soggetti vittime di irradiazioni accidentali, Riv. Med. Lav. Ig. Ind. 7, 31-42.

Pennarola R. (1984) Les techniques de la capillaroscopie dans le but de la prévention des sujets exposés aux radiations ionisantes. In Commun. VI Cong. Inter. IRPA, 7-12 maggio, Berlino, II, pp. 860-863.

Pennarola R., Perdereau B., Gongora R. (1991) La capillaroscopie en radiopathologie, Folia Med. 63 (1), $1-26$

Potten C.S. (1985) Radiation and skin. Taylor and Francis, London.

Rinaldi M. (1958) La séméiologia dei capillari sanguini. Calia, Napoli.

Turano L. (1930) Sulle modificazioni dei capillari alle radiazioni röentgen, Arch. Di Radiologia. 8, 349-363.

Vayssariat M., Priollet P. (1983) Atlas pratique de capillaroscopie. Revue de Médecine, Paris.

Willnow R. (1973) Biomicroscopes, Bibli. Anat. 11, 239-243.

Zweifach B.W. (1961) Functional behaviour of the microcirculation, I. Thomas, Springfield.

Zweifach B.W. (1973) Microcirculation, Ann. Rev. Physiol. 35, 117-150. 